\title{
Comparisons Between Infectious and Autoimmune Encephalitis: Clinical Signs, Biochemistry, Blood Counts, and Imaging Findings
}

This article was published in the following Dove Press journal: Neuropsychiatric Disease and Treatment

\author{
Chen-Na Huang (iD* \\ Xiao-Bing Tian (1D* \\ Shu-Min Jiang \\ Sheng-Hui Chang \\ Nan Wang \\ Ming-Qi Liu \\ Qiu-Xia Zhang \\ Ting Li \\ Lin-Jie Zhang \\ Li Yang
}

Department of Neurology, Tianjin Neurological Institute, Tianjin Medical University General Hospital, Tianjin 300052, People's Republic of China

*These authors contributed equally to this work
Correspondence: Li Yang

Tel +86-22-608I4536

Fax +86-22-608I747I

Email yangli200I@tmu.edu.cn
Objective: Infectious encephalitis (IE) and autoimmune encephalitis (AE) are symptomatically similar in clinic, however essentially different in pathogenesis. Therefore, the objective of this study was to identify specific features to distinguish the two types of encephalitis for early effective diagnosis and treatments through a comparative analysis.

Methods: Fifty-nine IE patients and 36 AE patients were enrolled. The patients with IE were divided into viral encephalitis (VE) and bacterial encephalitis (BE) according to the pathogens in cerebrospinal fluid (CSF). Patients with AE were categorized by with or without neural autoantibodies (NAAb). We further divided patients with NAAb into those with neural cell-surface antibodies (NSAbs) or intracellular antibodies (Abs). Clinical features, laboratory data, and imaging findings were compared between AE, IE, and subgroups. Results: Memory deficits, involuntary movement, and seizures were relatively more commonly presenting symptoms in AE patients $(\mathrm{p}<0.05)$. The positive rate of Pandy test was higher in IE patients $(\mathrm{p}=0.007)$. Decreased leukocyte, erythrocyte, and platelet counts in blood were found in IE patients $(\mathrm{p}<0.05)$. Lower serum calcium level was found in VE compared to BE $(\mathrm{p}=0.027)$. Meanwhile, higher serum calcium level was found in patients with NSAbs compared with intracellular Abs $(\mathrm{p}=0.034)$. However, higher levels of LDH in CSF were found in patients with intracellular Abs $(p=0.009)$. In magnetic resonance imaging, hippocampus lesions were more commonly present in patients with $\mathrm{AE}(\mathrm{p}=$ 0.042). Compared with AE patients, more IE patients displayed the background electroencephalogram rhythm of slow-frequency delta $(\mathrm{p}=0.013)$.

Conclusion: Involuntary movement and memory deficits were more specifically present in AE patients. CSF Pandy, blood routine test and hippocampus lesions detections were potential markers for distinguishing AE and IE. Further, CSF LDH, and serum calcium levels were potentially useful to distinguish subgroups of encephalitis.

Keywords: infectious encephalitis, autoimmune encephalitis, cell-surface antibodies, intracellular antibodies, imaging findings

\section{Introduction}

Encephalitis is caused by viral/bacterial infections, inflammation mediated by autoimmunity (acute disseminated encephalomyelitis (ADEM), Bickerstaff's brainstem encephalitis, and systemic autoimmune diseases), paraneoplastic syndrome, and other unclear causes. ${ }^{1}$ Infectious encephalitis (IE) and autoimmune encephalitis (AE) are symptomatically similar in clinic, however essentially different in pathogenesis. $^{2}$ They are difficult to distinguish, thus bringing ambiguities in the treatments and managements of AE and IE with similar symptoms, such as fever, 
seizures, psychiatric disorders, behavioral disorders, memory deficit, disturbance of consciousness, speech dysfunction, involuntary movement, focal neurologic deficit, ataxia and autonomic dysfunction. ${ }^{3,4}$ Virus can cross the blood-brain barrier and cause damage by direct virus mediated necrosis and indirect innate immune responses. ${ }^{5}$ The pathogenic bacteria induce neural damage by secreting virulence factors and inhibiting the host's immune response. ${ }^{6}$ Meanwhile, $\mathrm{AE}$ is caused by an antigenspecific cytotoxic $\mathrm{T}$ cells reaction or specific immune response mediated by antibodies (Abs). ${ }^{7}$ Since the discovery of anti-NMDAR antibody in $2007,{ }^{8}$ different types of neural autoantibodies (NAAb) including neural cellsurface antibodies (NSAbs) and Intracellular Abs have been identified. $^{9}$

IE and $\mathrm{AE}$ demonstrate similarity in clinical features, however they are different in their pathogenesis, therefore differences in specific features and lesions found in Magnetic Resonance Imaging (MRI), electroencephalogram (EEG) and laboratory data are distinguishable by further studies. Polymerase Chain Reaction (PCR) has been widely used for the diagnosis of IE, especially viral encephalitis (VE), although a negative test result does not exclude the diagnosis of encephalitis. ${ }^{10}$ Cerebrospinal fluid (CSF) culture is extremely crucial in patients with bacterial encephalitis (BE) in order to determine the type of pathogens. Techniques such as immunoblotting, cell-based assays (CBA), immunochemistry and indirect immunofluorescence (IIF) assays can be used to identify highly specific autoantibodies responsible for AE. ${ }^{11,12}$ Very different treatment plans are often applied to $\mathrm{AE}$ and IE patients, therefore, effective early identification and intervention is critical. In our study, we retrospectively analyzed the clinical characteristics, laboratory examinations, and imaging findings of IE and AE, in order to explore key features that potentially contribute to early diagnosis of such diseases, therefore benefiting the patients through appropriate treatment applications.

\section{Materials and Methods}

\section{Definitions and Criteria}

Clinical data for 59 patients with IE and 36 patients with AE were retrospectively collected at the Department of Neurology of Tianjin Medical University General Hospital from March 2009 to May 2020. Patients with AE met the following diagnostic criteria for definite AE with NAAb: ${ }^{1}$ 1) subacute onset of working memory deficits, altered mental status, or psychiatric symptoms; 2) at least one of the following: a) new focal central nervous system (CNS) findings; b) seizures not explained by a previously known seizure disorder; c) CSF white blood cell (WBC) counts $\geq$ $5 / \mathrm{mm}^{3}$; d) MRI features suggestive of encephalitis; 3 ) serum and/or CSF samples were positive for NAAb, at least one of the following: NSAbs: NMDA-receptor (R)antibody (Ab) (N-methyl-D-aspartate), CASPR2-R-Ab (contactin-associated protein 2), AMPA-R-Ab (2-amino3- (3-hydroxy-5-methyl-isoxazol-4-yl) propanoic acid), LGI1-R-Ab (leucine-rich glioma inactivated 1), GABAB$\mathrm{R}-\mathrm{Ab}$ (gamma-aminobutyric acid); Intracellular Abs: antiGAD65 (glutamic acid decarboxylase), anti-CV2 (CRMP5), anti-PNMA2 (Ma2/Ta), anti-Hu (ANNA1), anti-Ri (ANNA2), anti-Yo (PCA-1), anti-Amphiphysin.

In addition, other patients with $\mathrm{AE}$ met the following diagnostic criteria for definite AE without NAAb: ${ }^{1}$ 1) subacute onset of working memory deficits, seizures, or psychiatric symptoms suggesting involvement of the limbic system; 2) bilateral brain abnormalities on T2weighted imaging (T2WI) or fluid attenuated inversion recovery (FLAIR) of MRI highly restricted to the medial temporal lobes. 3) At least one of the following: a) CSF WBC counts $\geq 5 / \mathrm{mm}^{3}$; b) EEG with epileptic or slowwave activity involving the temporal lobes. 4) Serum and CSF samples were negative for NAAb. ADEM, Bickerstaff's brainstem encephalitis, and various $\mathrm{Ab}$ associated immunological systemic diseases, such as systemic lupus erythematosus were excluded from this study.

Patients who met the Consensus Statement of the IE Consortium reported in 2013 were enrolled in the IE groups. The Consensus include: ${ }^{13} 1$ ) altered mental status lasting > 24h; 2) at least 2 of the following: a) fever $\geq 38^{\circ}$ $\mathrm{C}$ within the $72 \mathrm{~h}$ before or after presentation; b) generalized or partial seizures; c) new onset of focal neurologic findings; d) CSF WBC counts $\geq 5 / \mathrm{mm}^{3}$, e) abnormal imaging findings consistent with encephalitis, f) abnormal EEG finding consistent with encephalitis; 3) CSF samples were tested by Real-Time quantitative Polymerase Chain Reaction (RT-qPCR) and bacteria culture and found at least one pathogen which was strongly associated with IE patients with positive CSF bacteria culture and definite drug susceptibility tests were included in BE group. Patients with positive viral nucleic acid were included in VE group. Patients with infections such as mycobacterium tuberculosis, parasites and fungi were excluded from this study. 


\section{Sample Collection and Laboratory Assays}

The CSF of all patients was collected when virus infection-like symptoms $\left(\mathrm{T}>38^{\circ} \mathrm{C}\right.$, headache, etc) were present. DNA sequences were extracted from samples and amplified by Applied Biosystems ${ }^{\text {TM }} 7500$ RT-qPCR instrument (Thermo Fisher Scientific, USA). The CSF of all patients was collected when bacterial infection-like symptoms ( $\mathrm{T}>38^{\circ} \mathrm{C}$, neck stiffness, etc) were present, and transferred to different culture bottles (Bactec Plus Aerobic/F, Bactec Lytic/10 Anaerobic/F, Bactec Peds Plus/F) (Becton, Dickinson and Company Sparks, MD 21152 USA) immediately, and underwent etiology inspection by Bacrec ${ }^{\mathrm{TM}}$ FX automated blood culture system (Becton, Dickinson and Company, USA) in microbiological laboratory. All serum and CSF samples from patients with AE were evaluated for NSAbs by IIF kits (Euroimmun, Lübeck, Germany) and CBA kits (Euroimmun, Lübeck, Germany) according to the manufacturer's instructions. All patients underwent serum and CSF laboratory tests, including all of the following: CSF examinations included: Pandy test, intracranial pressure, WBC counts, protein levels, chloride, glucose, lactate dehydrogenase (LDH), lactic acid (Lac), adenosine deaminase (ADA) and C-reactive protein (CRP). Blood examinations included: leukocyte, erythrocyte and platelet counts, and the levels of sodium, potassium, calcium, and chlorine.

\section{MRI and EEG Data Acquisition}

MRI data were acquired using a 3.0-Tesla MR system (Discovery MR750, General Electric, Milwaukee, WI, USA). All but two of the patients had brain MRI. The T1-weighted imaging (T1WI), T2WI, FLAIR and diffusion weighted imaging (DWI) of brain MRI were recorded. Seventy-seven (81\%) patients underwent a $24-$ hour EEG recording (10-20 system according to international EEG convention). EEG data were classified according to their background waves (alpha, beta, delta, theta), epileptiform activity (sharp waves, spike waves, sharp (or spike) slow wave complexes, both), slow waves activity (delta and/or theta activity), and localization (frontal region, temporal region, other regions). MRI and EEG were interpreted by skilled radiologists and electroencephalographers.

\section{Clinical Feature Scores}

The degree of disability was graded as per the modified Rankin Scale (mRS). A mild case was defined as mRS score of $0-2$ and a severe case as mRS score of 3-6. The level of consciousness was measured by the Glasgow Coma Score (GCS). Neurological functions for all patients were evaluated by 2 qualified neurologists according to the mRS and GCS at peak stage of diseases.

\section{Statistical Analysis}

The Statistical Package for the Social Sciences (SPSS 22.0) was used for statistical analysis. Figures were produced by GraphPad Prism Version 6. MannWhitney $U$-test was used for comparison of continuous variables and shown as mean $\pm \mathrm{SD}$, and the Pearson chisquared test or Fisher's exact test was used for comparison of categorical variables shown as counts (percentages). A $\mathrm{p}$ value $<0.05$ was considered statistically significant.

\section{Results}

\section{The Abs of Patients with $A E$ and the Pathogens of Patients with IE}

The Abs of patients with AE $(n=36)$ and the pathogens of patients with IE $(n=59)$ were shown in Figure 1 . Of 36 patients with $\mathrm{AE}, \mathrm{Abs}$ were detected in 28 patients. Of the 59 patients with IE, 16 patients were infected with virus, 38 patients were infected with bacteria, and 5 patients had double infections.

\section{The Serum and CSF Abs Titers of Patients with $A E$}

Positive anti-NMDA-R Ab was detected in 11 CSF samples and 4 serum samples from 11 AE patients. Positive anti-LGI1 Ab was detected in 9 CSF samples and 8 serum samples from $10 \mathrm{AE}$ patients. Positive anti-GABAB-R Ab was detected in 2 CSF samples and 2 serum samples of 2 patients. However, due to the update of detection technologies, abs titers were applied to only part of the patients, therefore the abs titer results were unknown for some of the patients (Figure 2). Patients with intracellular Abs were only obtained positive or negative results by CBA. All CSF samples and 3 serum samples from 6 patients had detection of positive intracellular Abs.

\section{Clinical Characteristics and Laboratory Tests of Patients with IE and AE}

As shown in Table 1, no significant differences were found in the onset age and gender between the two 


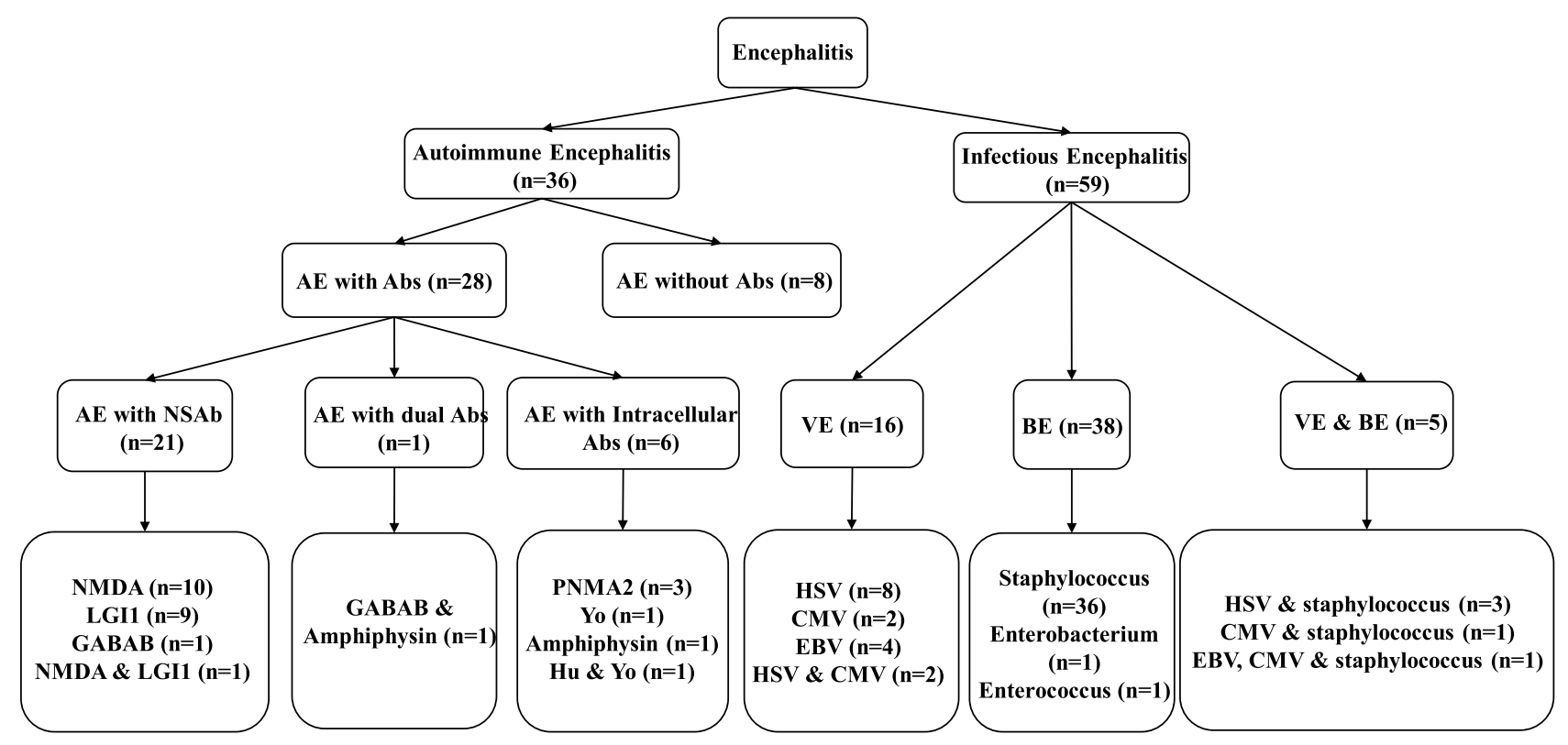

Figure I The antibodies of patients with $\mathrm{AE}$ and the pathogens of patients with IE.

Abbreviations: AE, autoimmune encephalitis; IE, infectious encephalitis; VE, viral encephalitis; BE, bacterial encephalitis; NSAbs, neuronal cell-surface antibodies; Abs, antibodies; NMDAR, N-methyl-d-aspartate receptor; LGII, leucine rich glioma inactivated-I; GABAB, gamma-aminobutyric acid B; PNMA2, paraneoplastic antigen MA2; HSV, herpes simplex virus; CMV, cytomegalovirus; EBV, Epstein-Barr virus; \&, and; n, number.

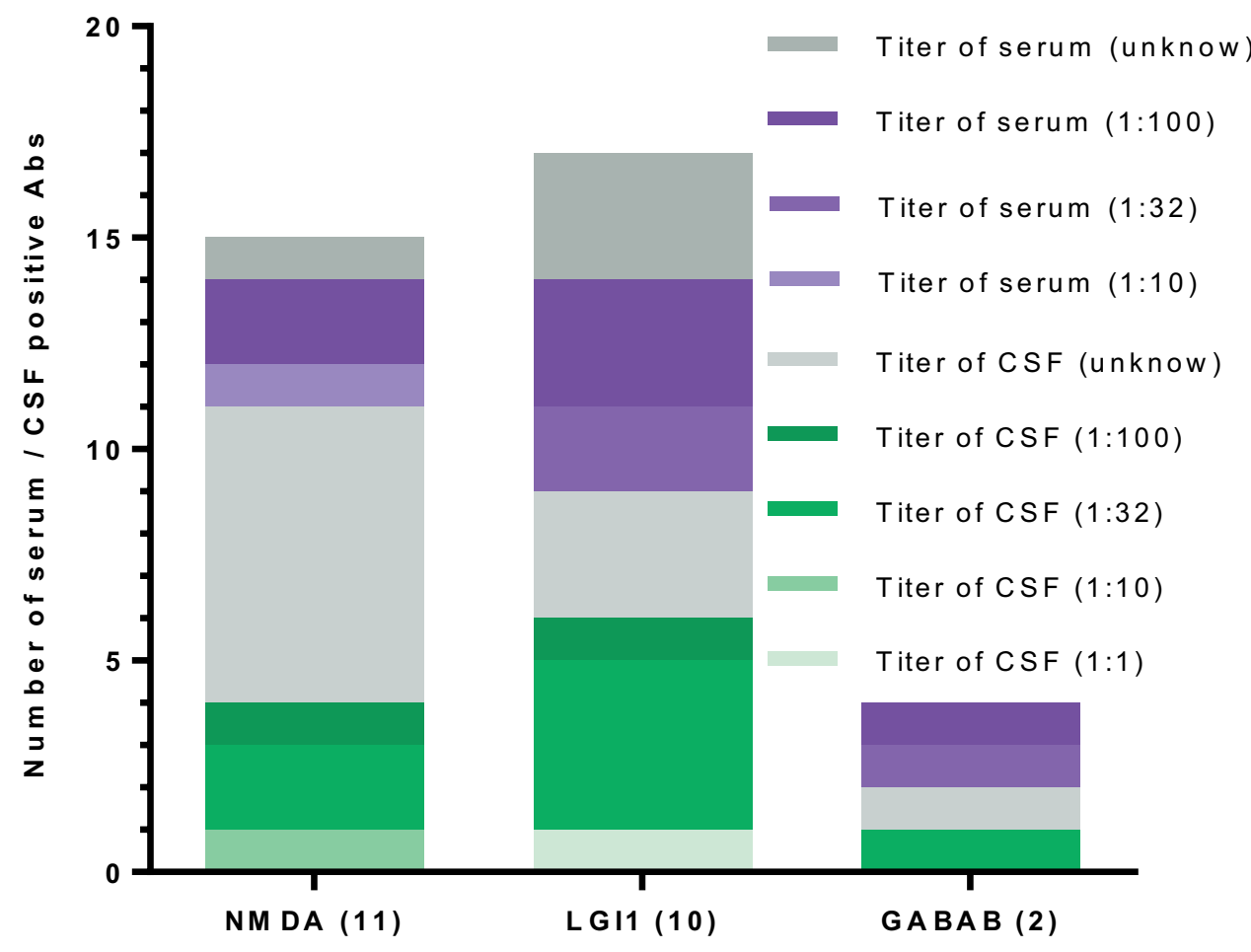

Figure 2 Abs titers of serum and CSF in AE patients with NSAbs.

Abbreviations: CSF, cerebral spinal fluid; Abs, antibodies; NMDAR, N-methyl-d-aspartate receptor; LGII, leucine rich glioma inactivated-I; GABAB, gamma-aminobutyric acid B; AE, autoimmune encephalitis; NSAbs, neural cell-surface antibodies.

diseases $(\mathrm{p}=0.160 ; \mathrm{p}=0.112)$. Headache and fever were more likely to occur in patients with IE $(p=$
$0.002 ; \mathrm{p}=0.006$ ). Involuntary movement, memory deficits, and seizure activity were more often observed 
Table I Clinical Characteristics and Laboratory Results of Patients with AE and IE

\begin{tabular}{|c|c|c|c|}
\hline & $\operatorname{IE}(n=59)$ & $\operatorname{AE}(n=36)$ & $\mathbf{p}$ \\
\hline Onset age (years) & $42.5 \pm 18$ & $47.1 \pm 15.9$ & 0.160 \\
\hline Female sex, No. (\%) & $26(44.1 \%)$ & $10(27.8 \%)$ & 0.112 \\
\hline Premonitory symptom, No. (\%) & $24(40.7 \%)$ & $9(25.0 \%)$ & 0.119 \\
\hline \multicolumn{4}{|l|}{ Onset symptom, No. (\%) } \\
\hline Headache & $30(50.8 \%)$ & $6(18.2 \%)$ & $0.002^{*}$ \\
\hline Fever & $40(67.8 \%)$ & $14(38.9 \%)$ & $0.006 *$ \\
\hline Psychiatric and behavior disorders & $25(42.4 \%)$ & $21(58.3 \%)$ & 0.131 \\
\hline Seizure & $25(42.4 \%)$ & $23(63.9 \%)$ & $0.042 *$ \\
\hline Decreased consciousness & $32(54.2 \%)$ & $20(55.6 \%)$ & 0.900 \\
\hline Speech dysfunction & $15(25.4 \%)$ & $10(27.8 \%)$ & 0.800 \\
\hline Involuntary movement & $2(3.4 \%)$ & $\mathrm{II}(30.6 \%)$ & $0.00 I^{*}$ \\
\hline Autonomic dysfunction & $5(8.5 \%)$ & $8(22.2 \%)$ & 0.113 \\
\hline Ataxia & $4(6.8 \%)$ & $3(8.3 \%)$ & 1.000 \\
\hline Memory deficit & $5(8.5 \%)$ & $13(36.1 \%)$ & $0.001 *$ \\
\hline Sleep disorders & $9(15.3 \%)$ & $8(22.2 \%)$ & 0.390 \\
\hline Focal neurologic deficit & $12(20.3 \%)$ & $9(25.0 \%)$ & 0.595 \\
\hline Pulmonary infection complications & $18(30.5 \%)$ & $10(27.8 \%)$ & 0.777 \\
\hline Tumor, No. (\%) & $3(5.15 \%)$ & $4(11.1 \%)$ & 0.493 \\
\hline Elapsed time between symptom onset and diagnosis (days) & $7.2 \pm 7.5$ & $33.9 \pm 48.7$ & $<0.001 * *$ \\
\hline Admission to hospital (days) & $16.9 \pm 8.4$ & $20.7 \pm 9.2$ & $0.011 *$ \\
\hline ICU stay, No. (\%) & $17(28.8 \%)$ & $5(13.9 \%)$ & 0.094 \\
\hline \multicolumn{4}{|l|}{ Immunotherapy, No. (\%) } \\
\hline Glucocorticoid & $15(25.4 \%)$ & $24(66.7 \%)$ & $<0.001 * *$ \\
\hline IVIG & $2(3.4 \%)$ & $8(22.2 \%)$ & $0.010 *$ \\
\hline \multicolumn{4}{|l|}{ Scores (at peak stage) } \\
\hline mRS 0-2, No. (\%) & $24(40.7 \%)$ & $14(38.9 \%)$ & 0.863 \\
\hline mRS 3-6, No. (\%) & $35(59.3 \%)$ & $22(61.1 \%)$ & 0.863 \\
\hline GCS & $11.2 \pm 4.1$ & $11.8 \pm 3.5$ & 0.770 \\
\hline \multicolumn{4}{|l|}{ The first CSF findings } \\
\hline Intracranial hypertension $(\mathrm{mmH} 2 \mathrm{O})$ & $183.1 \pm 68.3$ & $168.2 \pm 67.5$ & 0.193 \\
\hline CSF WBC (> $\left.5 \times 10^{6} / \mathrm{L}\right)$, No. (\%) & $35(59.3 \%)$ & $23(63.9 \%)$ & 0.658 \\
\hline CSF protein level $(g / L)$ & $0.6 \pm 0.4$ & $0.5 \pm 0.4$ & 0.086 \\
\hline CSF chloride level (mmol/L) & $124.2 \pm 6.7$ & $122.8 \pm 13.4$ & 0.890 \\
\hline CSF LDH level (U/L) & $34.9 \pm 36.2$ & $27 \pm 27.9$ & 0.224 \\
\hline CSF Lac level (mmol/L) & $2.3 \pm 1.5$ & $1.9 \pm 1.4$ & 0.210 \\
\hline CSF ADA level (U/L) & $1.5 \pm 1.7$ & $1.1 \pm 1.8$ & 0.130 \\
\hline CSF CRP level (mg/L) & $0.2 \pm 1.0$ & $0.1 \pm 0.1$ & 0.692 \\
\hline CSF/Blood glucose ratio $(<0.5)$, No. (\%) & $23(39.0 \%)$ & $13(36.1 \%)$ & 0.780 \\
\hline Positive rate of Pandy test, No. (\%) & $48(81.4 \%)$ & $20(44.4 \%)$ & $0.007^{*}$ \\
\hline \multicolumn{4}{|l|}{ The first blood findings } \\
\hline Blood sodium level (mmol/L) & $137.8 \pm 5.6$ & $137.3 \pm 5$ & 0.346 \\
\hline Blood potassium level (mmol/L) & $3.7 \pm 0.4$ & $3.9 \pm 0.4$ & 0.168 \\
\hline Blood calcium level (mmol/L) & $2.16 \pm 0.22$ & $2.20 \pm 0.16$ & 0.058 \\
\hline Blood chlorine level (mmol/L) & $99.7 \pm 4.7$ & $97.2 \pm 17$ & 0.859 \\
\hline Blood leukocyte count $\left(\times 10^{9} / \mathrm{L}\right)$ & $7.5 \pm 2.8$ & $10.3 \pm 3.6$ & $<0.00 I^{* *}$ \\
\hline Blood erythrocyte count $\left(\times 10^{12} / \mathrm{L}\right)$ & $4.1 \pm 0.7$ & $4.6 \pm 0.6$ & $0.001 *$ \\
\hline Blood platelet count $\left(\times 10^{9} / \mathrm{L}\right)$ & $227 \pm 82.8$ & $277.1 \pm 118.4$ & $0.034 *$ \\
\hline
\end{tabular}


Table I (Continued).

\begin{tabular}{|c|c|c|c|}
\hline & $\operatorname{IE}(n=59)$ & $\operatorname{AE}(n=36)$ & $\mathbf{p}$ \\
\hline \multicolumn{4}{|l|}{ The MRI findings } \\
\hline Hippocampus, No. (\%) & $13(22.0 \%)$ & $15(41.7 \%)$ & $0.042 *$ \\
\hline Amygdala, No. (\%) & $12(20.3 \%)$ & $12(33.3 \%)$ & 0.157 \\
\hline Memory deficit and hippocampus, No. (\%) & $\mathrm{I}(\mathrm{I} .7 \%)$ & $6(16.7 \%)$ & $0.011 *$ \\
\hline Memory deficit and amygdala, No. (\%) & $\mathrm{I}(\mathrm{I} .7 \%)$ & $6(16.7 \%)$ & $0.011 *$ \\
\hline \multicolumn{4}{|l|}{ The EEG findings } \\
\hline Alpha rhythm predominate, No. (\%) & $22(48.9 \%)$ & $17(53.1 \%)$ & 0.714 \\
\hline Beta rhythm predominate, No. (\%) & $5(\mid 1.1 \%)$ & $2(6.3 \%)$ & 0.693 \\
\hline Delta rhythm predominate, No. (\%) & $19(42.2 \%)$ & $5(15.6 \%)$ & $0.013^{*}$ \\
\hline Thet rhythm predominate, No. (\%) & $20(44.4 \%)$ & $10(31.3 \%)$ & 0.242 \\
\hline Delta and/or theta activity, No. (\%) & $22(48.9 \%)$ & $17(53.1 \%)$ & 0.714 \\
\hline Sharp wave, No. (\%) & $12(26.7 \%)$ & $12(37.5 \%)$ & 0.312 \\
\hline Spike wave, No. (\%) & $\mathrm{I}(2.2 \%)$ & $4(12.5 \%)$ & 0.154 \\
\hline Sharp-slow complex wave, No. (\%) & $6(13.3 \%)$ & $4(12.5 \%)$ & 1.000 \\
\hline Spike-slow wave, No. (\%) & $5(11.1 \%)$ & $\mathrm{I}(3.1 \%)$ & 0.391 \\
\hline Frontal region predominate, No. (\%) & $14(31.1 \%)$ & $15(46.9 \%)$ & 0.159 \\
\hline Temporal region predominate, No. (\%) & $8(17.8 \%)$ & $10(31.3 \%)$ & 0.169 \\
\hline Other regions, No. (\%) & $4(8.9 \%)$ & $7(21.9 \%)$ & 0.185 \\
\hline
\end{tabular}

Notes: Data are presented as No. (\%) or mean \pm standard deviation; significant differences are indicated by *p $<0.05$; $* * p<0.00$ I.

Abbreviations: AE, autoimmune encephalitis; IE, infectious encephalitis; No., number; CSF, cerebral spinal fluid; mRS, modified Rankin Scale; GCS, Glasgow Coma Score; IVIG, intravenous immunoglobulin; WBC, white blood cell; ICU, intensive care unit; CRP, C-reactive protein; LDH, lactate dehydrogenase; Lac, lactic acid; ADA, adenosine deaminase; MRI, magnetic resonance imaging; EEG, electroencephalogram.

in AE patients ( $\mathrm{p}=0.001 ; \mathrm{p}=0.001 ; \mathrm{p}=0.042)$. The day of admission to hospital $(\mathrm{p}=0.011)$ and the time from symptom onset to diagnosis $(p<0.001)$ were longer in AE patients. However, other symptoms were similar between the two groups. No significant differences were found in mRS and GCS of AE and IE patients. Higher positive rate of Pandy test was found in IE patients than AE patients $(p=0.007)$. Lower leukocyte, erythrocyte, and platelet counts were found in IE patients than AE patients ( $\mathrm{p}<0.001$; $\mathrm{p}=0.001$; $\mathrm{p}=0.034)$. For the clinical treatments, $54(91.5 \%)$ patients with IE received anti-infective therapies, while 15 (25.4\%) IE patients received corticosteroids. The majority (66.7\%) of AE patients received corticosteroids, $8(22.2 \%)$ AE patients received intravenous immunoglobulin, and only $1(2.8 \%)$ AE patient was treated with rituximab. The EEG information was collected and analyzed. The background waves of IE patients more frequently exhibited delta rhythm than AE patients $(p=0.013)$. Delta and/or theta activities were the most common abnormal wave in both IE (48.9\%) and AE patients (53.1\%) (Table 1).

\section{Subgroup Analysis of Clinical}

Characteristics and Laboratory Results: Comparisons of Patients with VE and BE; Differences of AE Patients with NSAbs, Intracellular Abs and NAAb Negative

We further divided patients with IE $(n=59)$ into VE group $(\mathrm{n}=16)$ and BE group $(\mathrm{n}=38)$. Five patients with double infection were excluded. Headache was more prevalent in the patients with BE compared to those with $\operatorname{VE}(\mathrm{p}=$ 0.027 ), and the levels of serum calcium were lower in patients with VE $(p=0.027)$. There were $21 \mathrm{AE}$ patients with NSAbs, 6 AE patients with Intracellular Abs and 8 AE patients without NAAb. Only 1 AE patient with double Abs (GABAB and Amphiphysin) was excluded. The levels of CSF LDH were higher in patients with intracellular Abs than NSAbs $(p=0.009)$. Levels of CSF protein, $\mathrm{LDH}, \mathrm{Lac}$ and ADA were higher in AE patients without NAAb than those with NSAbs $(p=0.010, p=0.048, p=$ $0.030, p=0.039$ ). As for blood tests, serum calcium levels were higher in AE patients with NSAbs than patients with intracellular Abs $(p=0.034)$. Patients with intracellular 
Abs presented significantly decreased levels of erythrocyte count in blood $(\mathrm{p}=0.007)$. Additionally, patients with NSAbs had significantly longer duration of hospital stays $(\mathrm{p}=0.021)$ (Table 2).

\section{Lesion Distribution of Brain MRI in Patients with AE and IE}

All patients underwent brain MRI examinations except for 2 patients: a young male and a female with unstable clinical conditions. Computed tomography (CT) scan of the brain was performed in those 2 IE patients and the imaging results were normal. Patients with AE were more likely associated with hippocampal lesions than IE patients $(p=0.042)$, they were also found more likely to suffer memory deficits $(p=0.010)$ (Table 1). Differences of lesion distribution between the two groups were shown in Figure 3. A total number of $30(52.6 \%)$ IE patients and 25 (69.4\%) AE patients demonstrated intracranial abnormal signal. Temporal lobe lesions were the most commonly found feature in brain MRI of 21 (70\%) IE patients and $20(80 \%)$ AE patients, however, no statistical significance was found between the two groups. Among these patients, 13 IE patients and 15 AE patients demonstrated hippocampal lesions. The second most common brain region with observed lesions was amygdaloid in both groups. Followed by that, frontal lobe in IE patients,

Table 2 Subgroup Analysis of Clinical Characteristics and Laboratory Results: Comparisons of Patients with VE and BE; Differences of AE Patients with NSAbs and Intracellular Abs

\begin{tabular}{|c|c|c|c|c|c|}
\hline & \multicolumn{2}{|l|}{ IE } & \multicolumn{3}{|l|}{ AE } \\
\hline & VE & BE & NSAbs & $\begin{array}{l}\text { Intracellular } \\
\text { Abs }\end{array}$ & $\begin{array}{l}\text { NAAb } \\
\text { negative }\end{array}$ \\
\hline \multicolumn{6}{|l|}{ Onset symptom, No. (\%) } \\
\hline Headache & $4(25.0 \%)$ & $22(57.9 \%) *$ & $3(14.3 \%)$ & $\mathrm{I}(\mathrm{I} 6.7 \%)$ & $2(25.0 \%)$ \\
\hline Fever & $13(81.3 \%)$ & $23(60.5 \%)$ & $6(28.6 \%)$ & $3(50.0 \%)$ & $5(62.5 \%)$ \\
\hline Seizure & $9(56.3 \%)$ & $13(34.2 \%)$ & $15(7 \mid .4 \%)$ & $3(50.0 \%)$ & $4(50.0 \%)$ \\
\hline Involuntary movement & $\mathrm{I}(6.3 \%)$ & $\mathrm{I}(2.6 \%)$ & $9(42.9 \%)$ & $\mathrm{I}(16.7 \%)$ & $\mathrm{I}(\mathrm{I} 2.5 \%)$ \\
\hline Memory deficit & $2(12.5 \%)$ & $3(7.9 \%)$ & II (52.4\%) & $\mathrm{I}(\mathrm{I} 6.7 \%)$ & $\mathrm{I}(\mathrm{I} 2.5 \%)$ \\
\hline Elapsed time between symptom onset and diagnosis (days) & $7.8 \pm 7.3$ & $7.2 \pm 8$ & $41.8 \pm 60.1$ & $38.7 \pm 31$ & $10.9 \pm 7.8^{\mathrm{b}}$ \\
\hline Admission to hospital (days) & $18.6 \pm 9.3$ & $15.9 \pm 8.4$ & $21.3 \pm 8.5$ & $15.2 \pm 3.7^{\#}$ & $23.5 \pm 12.8$ \\
\hline ICU stay, No. (\%) & $6(37.5 \%)$ & $10(26.3 \%)$ & $3(14.3 \%)$ & $\mathrm{I}(\mathrm{I} 6.7 \%)$ & $\mathrm{I}(\mathrm{I} 2.5 \%)$ \\
\hline \multicolumn{6}{|l|}{ The first CSF findings } \\
\hline CSF WBC (> $\left.5 \times 10^{6} / \mathrm{L}\right)$, No. (\%) & $8(50.0 \%)$ & $23(60.5 \%)$ & $13(61.9 \%)$ & $3(50.0 \%)$ & $7(87.5 \%)$ \\
\hline CSF protein level $(\mathrm{g} / \mathrm{L})$ & $0.7 \pm 0.6$ & $0.6 \pm 0.4$ & $0.4 \pm 0.1$ & $0.8 \pm 0.6$ & $0.7 \pm 0.5^{\mathrm{a}}$ \\
\hline CSF chloride level (mmol/L) & $124.1 \pm 5$ & $124.6 \pm 7.5$ & $125.4 \pm 6.2$ & $122.7 \pm 7.1$ & $114.9 \pm 25.6$ \\
\hline CSF LDH level (U/L) & $47.1 \pm 4 I .5$ & $29.8 \pm 35.3$ & $19.3 \pm 11.4$ & $49.2 \pm 58.6^{\#}$ & $32.9 \pm 19.7^{\mathrm{a}}$ \\
\hline CSF Lac level (mmol/L) & $2.4 \pm 1.8$ & $2.2 \pm 1.4$ & $1.6 \pm 0.4$ & $3.1 \pm 3.3$ & $1.9 \pm 0.3^{\mathrm{a}}$ \\
\hline CSF ADA level (U/L) & $1.5 \pm 1.6$ & $1.4 \pm 1.6$ & $0.8 \pm 1.5$ & $2.4 \pm 3.1$ & $1.2 \pm 1.1^{\mathrm{a}}$ \\
\hline CSF CRP level (mg/L) & $0.6 \pm 1.8$ & $0.1 \pm 0.2$ & $0.1 \pm 0.1$ & $0.1 \pm 0.1$ & $0.1 \pm 0$ \\
\hline Positive rate of Pandy test, No. (\%) & $12(75 \%)$ & $32(84.25 \%)$ & $10(47.6 \%)$ & $5(83.3 \%)$ & $5(62.5 \%)$ \\
\hline \multicolumn{6}{|l|}{ The first serum findings } \\
\hline Blood sodium level (mmol/L) & $137.8 \pm 5.2$ & $138 . \mid \pm 5.5$ & $136.4 \pm 4.9$ & $138.7 \pm 4.5$ & $138.7 \pm 5.7$ \\
\hline Blood potassium level (mmol/L) & $3.6 \pm 0.4$ & $3.8 \pm 0.5$ & $3.9 \pm 0.4$ & $3.7 \pm 0.5$ & $3.9 \pm 0.4$ \\
\hline Blood calcium level (mmol/L) & $2.1 \pm 0.2$ & $2.2 \pm 0.2^{*}$ & $2.2 \pm 0.2$ & $2.1 \pm 0.1^{\#}$ & $2.2 \pm 0.2$ \\
\hline Blood chlorine level (mmol/L) & $100.2 \pm 4.5$ & $99.7 \pm 4.6$ & $98.6 \pm 5.2$ & $102 \pm 4.6$ & $89.7 \pm 35.5$ \\
\hline Blood leukocyte count $\left(\times 10^{9} / \mathrm{L}\right)$ & $6.7 \pm 2.1$ & $7.7 \pm 3$ & $10.5 \pm 4$ & $8.9 \pm 3.2$ & $10.4 \pm 3$ \\
\hline Blood erythrocyte count $\left(\times 10^{12} / \mathrm{L}\right)$ & $3.8 \pm 1.1$ & $4.2 \pm 0.6$ & $4.7 \pm 0.6$ & $4 \pm 0.5^{\#}$ & $4.7 \pm 0.7$ \\
\hline Blood platelet count $\left(\times 10^{9} / \mathrm{L}\right)$ & $199.4 \pm 72.6$ & $236.3 \pm 89.1$ & $272 \pm 106$ & $248.7 \pm 90.2$ & $311.4 \pm 173.5$ \\
\hline
\end{tabular}

Notes: Data are presented as No. (\%) or mean \pm standard deviation; significant differences are indicated by *VE versus BE, $\mathrm{p}<0.05 ;{ }^{\#} \mathrm{NSAbs}$ versus Intracellular Abs, $\mathrm{p}<$ 0.05 ; ${ }^{a}$ NSAbs versus NAAb negative $p<0.05$; ' Intracellular Abs versus NAAb negative $p<0.05$.

Abbreviations: AE, autoimmune encephalitis; IE, infectious encephalitis; VE, viral encephalitis; BE, bacterial encephalitis; No., number; CSF, cerebral spinal fluid; mRS, modified Rankin Scale; GCS, Glasgow Coma Score; IVIG, intravenous immunoglobulin; WBC, white blood cell; ICU, intensive care unit; CRP, C-reactive protein; LDH, lactate dehydrogenase; Lac, lactic acid; ADA, adenosine deaminase; Abs, antibodies; NSAbs, neural cell-surface antibodies; NAAb, neural autoantibodies. 


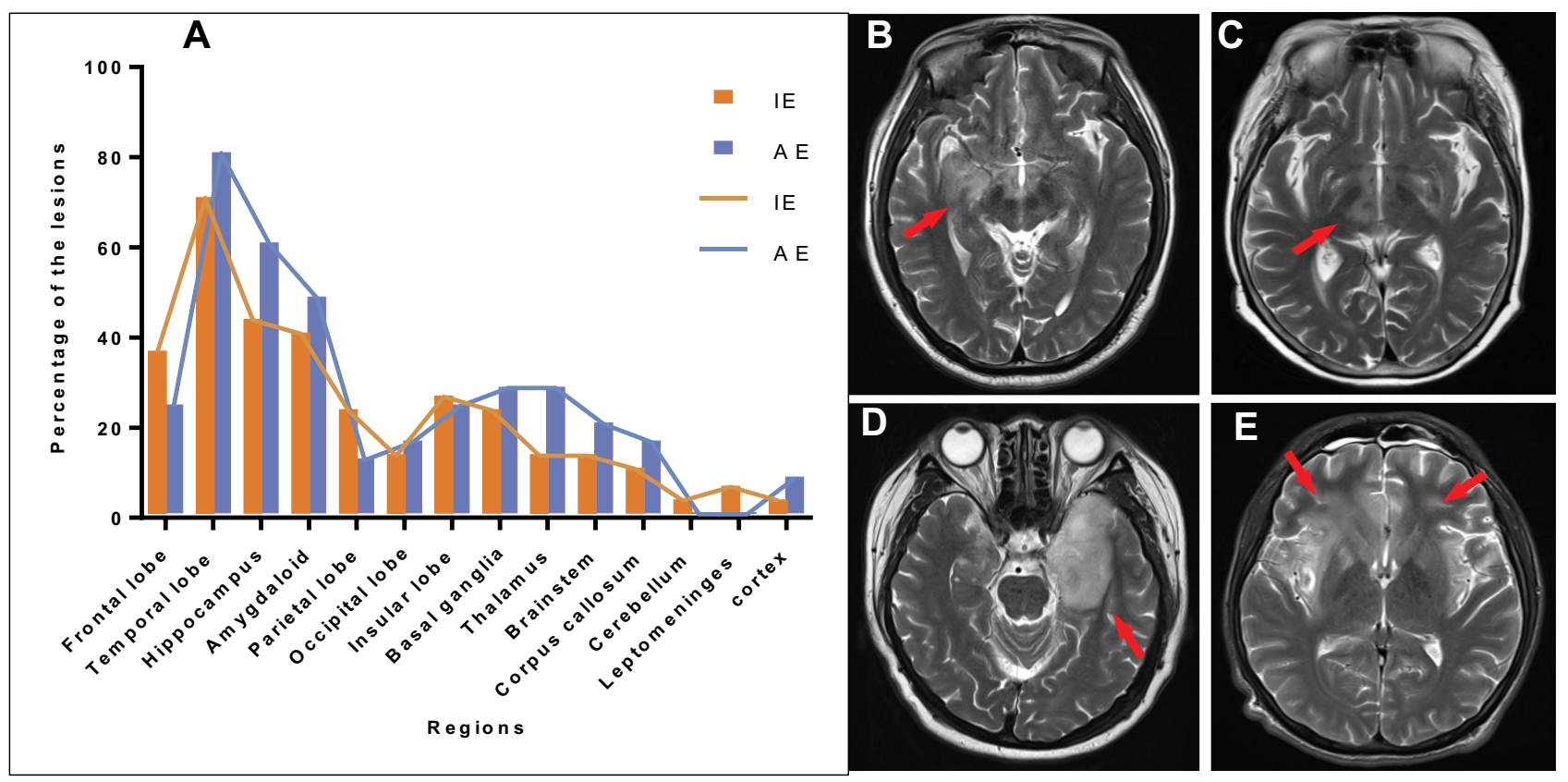

Figure 3 Lesions' distribution in brain MRI in patients with AE and IE, and abnormal MRI images in 4 patients. The lesions' distribution of 30 IE patients and 25 AE patients with abnormal MRI image (A). Brain MRIs show T2 hyperintensities relatively restricted to the unilateral medial temporal lobe in a patient with AE ((B), arrows). Panel (C) shows thalamus T2 hyperintensities in a patient with AE (arrow). Panel (D) shows unilateral temporal lobe T2 hyperintensities in a patient with IE (arrow). Panel (E) shows bilateral deep frontal lobe T2 hyperintensities in a patient with IE (arrow).

Abbreviations: $A E$, autoimmune encephalitis; IE, infectious encephalitis.

and both thalamus and basal ganglia in AE patients were also commonly damaged. Meanwhile, brain regions such as cerebellum, leptomeninges and cortex were the least common regions with observed lesions (Figure 3).

\section{Discussion}

IE and $\mathrm{AE}$ are symptomatically similar, however very different in terms of pathogenesis and clinical treatments. The symptoms, laboratory results, and MRI presentations were often confusing especially in patients with critical conditions. Therefore, accurate distinguishing of such diseases is crucial for patients with limited medical records at the early stages of the diseases.

Specific clinical presentations were the first step during diagnosis. Fever and headache were more commonly found in IE patients, while in subgroups of IE, BE patients were more prone to suffering headache than VE patients. Previous studies also reported that IE patients more frequently presented with fever and headache, ${ }^{2,14}$ which was consistent with our results. A large amount of literature demonstrated that meningitis was mediated by the activated human immune defence system caused by infective pathogen invasion. Meanwhile, bacteria also damaged meninges by producing bacterial toxins. The inflammation of meninges subsequently stimulated nerve roots and caused the headache. ${ }^{6,15}$ NMDAR include N-methylD-aspartate receptor 1 (NR1), NR2, and NR3 subunits, in which the mechanisms of headache were mainly associated with NR2 subunit. ${ }^{16}$ NR2 was over-expressed in synapses, where the terminations of nociceptive afferents were. ${ }^{17}$ In this study, a total of $6 \mathrm{AE}$ patients demonstrated headache, while 5 of them were found to present antiNMDAR antibodies. However, the detailed characteristics of the headache were not recorded in most of our patients. Hence, the differences in the feature of headache between $\mathrm{IE}$ and $\mathrm{AE}$ were not clear. For future studies, detailed data regarding headache would be needed. Furthermore, involuntary movement, memory deficits and seizures were found to be more prevalent in AE patients than IE patients. Manifestations of involuntary movement included repetitive rhythmic ocular, jaw, facial, lingual, limb and trunk movements, opisthotonus, and dystonic limb posturing. ${ }^{18}$ Fronto-striatal disinhibition, cortico-limbic, hypothalamus and brainstem disconnections, the dysfunctions of basal ganglia were associated with involuntary movement. ${ }^{19,20}$ A previous study found that the antibody-mediated NMDAR decreases inactivated GABA-ergic neurons, therefore leading to movement disorders. ${ }^{20}$ In our study, 6 out of 10 NMDAR-Abs positive patients demonstrated involuntary movement, while the major cause of 
movement disorders in patients with anti-Ma2 encephalitis was cytotoxic T-cell-mediated neurologic damage of basal ganglia. $^{21}$ Vigliani et al also found that a minority of patients with intracellular Abs presented with involuntary movement. $^{22}$ In this study, a total number of $11(30.6 \%)$ AE patients presented with involuntary movement, significantly higher than IE patients. Similarly, Hou et al found that $25(45.5 \%)$ AE patients presented with involuntary movement. ${ }^{23}$ Gable et al also reported that involuntary movement was more commonly found in AE patients $(20$ individuals, 63\%) than IE patients (3 individuals, 6\%). ${ }^{14}$

Atrophy of hippocampus was closely correlated with memory deficits. Hansen et al found that in different periods of the course of AE, 4/7 AE patients demonstrated varying degrees of atrophies of hippocampal volume, which was associated with the decline of verbal memory and figural memory. ${ }^{24}$ We also found that AE patients were more likely to present hippocampus lesions than IE patients. Patients with LGI1-Ab demonstrated memory disorders, due to the fact that the LGI1 gene transcripts were enriched in CA3 region of the hippocampus. ${ }^{25,26}$ In this study, 9 patients with anti-LGI1 encephalitis were enrolled, while 4 of them demonstrated memory deficits. The CA1 region of the hippocampus contained the highest density of NMDAR in the brain, and the internalization of NMDAR in CA1 region caused severe memory deficits. ${ }^{27}$ In this study, 6 out of 10 anti-NMDAR encephalitis patients demonstrated memory deficits. The hippocampus function was damaged due to the binding of Ma2 to cytoplasmic protein in the area of limbic system. ${ }^{28}$ Similarly, limbic system damage caused also be caused by anti-Hu and anti-CV2 autoimmunity, as those antigen expressions were exclusively limited within neurons. ${ }^{29}$ As mentioned previously, AE patients were more likely to suffer from memory impairments, while in our study, a small proportion of IE patients also demonstrated memory problems. Of the 4 IE patients with memory decline, 2 cases were infected with Herpes simplex virus (HSV). Previous research reported that HSV infection was associated with memory decline. Receptors of HSV for viral entry were expressed in the hippocampus thus making the hippocampus vulnerable to HSV infection. ${ }^{30}$ NAAbmediated inhibitory synaptic transmission decrease and intrinsic excitability increase were the triggers of epilepsy in AE patients. ${ }^{31}$ The loss of the LGI1 receptor expression inhibited AMPA receptor-mediated synaptic transmission, reduced AMPA receptor, and therefore caused seizures. ${ }^{32}$ There were 7/9 patients with LGI1-Abs who presented with seizures. Yeo et al also found that anti-LGI1 /CASPR2 seropositive patients were more likely to be associated with seizures. ${ }^{33}$ Our data demonstrated that AE patients $(63.9 \%)$ were more likely to develop seizures than those with IE (42.4\%). A similar study also found that epileptic seizures were more prevalent in $\mathrm{AE}$ patients $(88 \%)$ than in IE patients $(21 \%)^{2}$ Therefore, in clinic, it was of critical importance to detect specific autoantibodies related to AE for accurate diagnosis of patients who demonstrated symptoms such as involuntary movement, memory deficit, and seizures.

Previous research found the robust link between viral/ bacterial infections and the declined counts of leukocyte, erythrocyte, and platelet. ${ }^{6,34-36}$ Epstein-Barr virus was found to infect B-cells and provoke oligoclonal expansion of suppressor T-cells, which destroyed the hematopoiesis of bone marrow, and subsequently caused the decrease of erythrocyte count. The pathophysiology of decline in platelet count was found to be caused by the binding of Epstein-Barr virus to platelet, leading to the presence of platelet-destroying agglutinin. ${ }^{37}$ In this study, most BE patients were infected with bacterial staphylococcus, which produced cytolysins, including leukocidins and hemolysins, therefore causing decreases in the numbers of erythrocytes and leukocytes. ${ }^{6}$ Interestingly, the erythrocyte counts were decreased in AE patients with intracellular Abs compared to those with NSAbs. Intracellular Abs, also known as onconeuronal Abs, are associated with specific paraneoplastic syndrome. A previous study found that patients with intracellular Abs often presented with poor response to immunotherapy and unfavorable prognosis. ${ }^{11}$ However, the underlying mechanisms of erythrocyte damage in intracellular Abs-positive individuals remain unclear.

CSF data were an important reference in the determination of the causes of encephalitis. The Pandy test was a qualitative method to quickly detect abnormal protein concentrations in CSF by precipitation, and globulins played an important role in the process of precipitation. The increased globulin in CSF was caused by the activation of immune and defence system by foreign invaders, such as bacteria and virus, and subsequent positive Pandy test. $^{38}$ We found that the positive rates of Pandy tests were higher in IE patients than AE patients. CNS diseases, such as cerebral infarction, hemorrhage, infections and prolonged seizures, are also associated with increases in the levels of CSF LDH. ${ }^{39}$ The CSF LDH was significantly higher in $\mathrm{AE}$ patients with intracellular Abs than those 
with NSAbs. This was potentially due to the cytotoxic T-cells-mediated neural damage and death of AE patients with Intracellular Abs. ${ }^{40}$ Elishkevitz et al reported that the damage of neurons caused the rise of CSF LDH in patients with multiple sclerosis. ${ }^{41}$ Likewise, the levels of CSF protein, $\mathrm{LDH}, \mathrm{Lac}$ and ADA were found to be higher in AE patients without NAAb than those with NSAbs. However, the potential causes were still not well understood, and worthful for further studies.

In this study, the serum calcium levels were found to be lower in VE patients than BE patients. HSV or CMV infections induced the activation of Akt-mediated signaling pathway, which was a modulator of calcium influx. In turn, the activation of Akt promoted HSV or CMV entry. Calcium influx elevation caused decreased serum calcium. $^{42,43}$ The calcium entry into intracellular via NMDAR was activated by the Akt-mediated signaling pathway, and the disturbance of NMDAR blocked the calcium influx in patients with anti-NMDAR encephalitis. ${ }^{44}$ The disruption of LGI1 receptor decreased the expression of AMPA receptor, a major mediator of calcium influx. ${ }^{45}$ Collectively, the levels of serum calcium were increased due to reduced calcium influx in $\mathrm{AE}$ patients with NSAbs.

Detection of brain lesions by MRI scans revealed that temporal lobe lesions were the most common abnormal imaging feature in both types of encephalitis. Consistent with previous studies, it was suggested that lesions in the medial temporal lobes and the hippocampus were often involved in AE and HSV encephalitis patients. In this study, 8 out of 13 patients with HSV infection demonstrated hippocampal lesions. As revealed by EEG results, more IE patients presented with increases in delta background waves than $\mathrm{AE}$ patients. ${ }^{46-48}$ The slow-frequency wavebands delta usually appeared during the night. Viral infection of CNS caused increases of delta rhythm presented with circadian rhythm dysfunction. ${ }^{49}$

Taken together, our study suggested that the combination of symptomatic differences was potentially useful for preliminary distinguishing of $\mathrm{AE}$ and $\mathrm{IE}$ in clinic. Followed by that, further distinguishing was performed between AE patients with NSAbs and those with Intracellular Abs, and between $\mathrm{VE}$ and $\mathrm{BE}$, according to the key differences. This two-stepped diagnosis strategy was potentially useful for accurate and effective early diagnosis, therefore targeted therapies could be applied in time. However, this study had a retrospective single-center design and was therefore limited. Thus, multicenter and maximus sample clinical analysis iare necessary in further studies.

\section{Conclusion}

In conclusion, in this study, we found that AE patients were more likely to be associated with involuntary movement, memory deficits, and seizures. As revealed by CSF detections, IE patients demonstrated higher positive rates in Pandy tests, while AE patients with intracellular Abs demonstrated higher levels of LDH than those with NSAbs. In addition, serum erythrocyte and platelet counts were found to be decreased in IE patients. The serum calcium levels were found to be lower in patients with VE than $\mathrm{BE}$, and higher in patients with NSAbs than intracellular Abs. The hippocampal lesions revealed by MRI and EEG delta background rhythm were more commonly found in AE patients. All these features gave a clue for distinguishing $\mathrm{AE}$ and $\mathrm{IE}$ patients, and their respective subgroups. Further imaging analyses were potentially useful in differential diagnosis of these two types of encephalitis.

\section{Ethical Approval}

The study was approved by the Ethics Committee of Tianjin Medical University General Hospital (Ethical NO.IRB2020-WZ-113) and all the participants provided informed consent. The participants were informed about the purpose of the study, and that it was conducted in accordance with the Declaration of Helsinki.

\section{Acknowledgments}

We are extremely grateful to clinicians who assessed the severity of the disease, and acknowledge the efforts of researchers who worked on the clinical and neuroimaging data collection. We also would like to thank all patients for their participation in this study.

\section{Funding}

This work was supported by the National Natural Science Foundation of China (grant number: 81771363) and the National Science Foundation of Tianjin (grant number: 18JCQNJC81700).

\section{Disclosure}

The authors report no conflicts of interest in this work. 


\section{References}

1. Graus F, Titulaer MJ, Balu R, et al. A clinical approach to diagnosis of autoimmune encephalitis. Lancet Neurol. 2016;15(4):391-404. doi:10.1016/S1474-4422(15)00401-9

2. Wagner JN, Kalev O, Sonnberger M, Krehan I, von Oertzen TJ. Evaluation of clinical and paraclinical findings for the differential diagnosis of autoimmune and infectious encephalitis. Front Neurol. 2018;9:434. doi:10.3389/fneur.2018.00434

3. Quist-Paulsen E, Kran AB, Lindland ES, et al. To what extent can clinical characteristics be used to distinguish encephalitis from encephalopathy of other causes? Results from a prospective observational study. BMC Infect Dis. 2019;19(1):80. doi:10.1186/s12879-0183570-2

4. Hermetter C, Fazekas F, Hochmeister S. Systematic review: syndromes, early diagnosis, and treatment in autoimmune encephalitis. Front Neurol. 2018;9:706. doi:10.3389/fneur.2018.00706

5. Stahl JP, Mailles A. Herpes simplex virus encephalitis update. Curr Opin Infect Dis. 2019;32(3):239-243. doi:10.1097/QCO.000000000 0000554

6. Olchowik-Grabarek E, Sekowski S, Bitiucki M, et al. Inhibition of interaction between Staphylococcus aureus $\alpha$-hemolysin and erythrocytes membrane by hydrolysable tannins: structure-related activity study. Sci Rep. 2020;10(1):11168. doi:10.1038/s41598-020-68030-1

7. Dalmau J. [Limbic encephalitis and variants related to neuronal cell membrane autoantigens]. Rinsho Shinkeigaku $=$ Clinical Neurology 2008;48(11):871-874. doi:10.5692/clinicalneurol.48.871

8. Dalmau J, Tüzün E, Wu HY, et al. Paraneoplastic anti-N-methylD-aspartate receptor encephalitis associated with ovarian teratoma. Ann Neurol. 2007;61(1):25-36. doi:10.1002/ana.21050

9. Lancaster E. The diagnosis and treatment of autoimmune encephalitis. J Clin Neurol. 2016;12(1):1-13. doi:10.3988/jcn.2016. 12.1.1

10. Tunkel AR, Glaser CA, Bloch KC, et al. The management of encephalitis: clinical practice guidelines by the infectious diseases society of America. Clin Infect Dis. 2008;47(3):303-327. doi:10.1086/ 589747

11. Ricken G, Schwaiger C, De Simoni D, et al. Detection methods for autoantibodies in suspected autoimmune encephalitis. Front Neurol. 2018;9:841. doi:10.3389/fneur.2018.00841

12. Dalmau J, Gleichman AJ, Hughes EG, et al. Anti-NMDA-receptor encephalitis: case series and analysis of the effects of antibodies. Lancet Neurol. 2008;7(12):1091-1098. doi:10.1016/S1474-4422(08) 70224-2

13. Venkatesan A, Tunkel AR, Bloch KC, et al. Case definitions, diagnostic algorithms, and priorities in encephalitis: consensus statement of the international encephalitis consortium. Clin Infect Dis. 2013;57 (8):1114-1128. doi:10.1093/cid/cit458

14. Gable MS, Sheriff H, Dalmau J, Tilley DH, Glaser CA. The frequency of autoimmune N-methyl-D-aspartate receptor encephalitis surpasses that of individual viral etiologies in young individuals enrolled in the California encephalitis project. Clin Infect Dis. 2012;54(7):899-904. doi:10.1093/cid/cir1038

15. Tamune H, Kuki T, Kashiyama T, Uchihara T. Does this adult patient with jolt accentuation of headache have acute meningitis? Headache. 2018;58(10):1503-1510. doi:10.1111/head.13376

16. Ma C, Wang C, Zhang Q, Lian Y. Emerging role of prodromal headache in patients with anti-N-methyl-D-aspartate receptor encephalitis. J Pain Res. 2019;12:519-526. doi:10.2147/JPR.S18 9301

17. Nagy GG, Watanabe M, Fukaya M, Todd AJ. Synaptic distribution of the NR1, NR2A and NR2B subunits of the N-methyl-d-aspartate receptor in the rat lumbar spinal cord revealed with an antigen-unmasking technique. Eur $J$ Neurosci. 2004;20(12):33 01-3312. doi:10.1111/j.1460-9568.2004.03798.x
18. Kleinig TJ, Thompson PD, Matar W, et al. The distinctive movement disorder of ovarian teratoma-associated encephalitis. Mov Disord. 2008;23(9):1256-1261. doi:10.1002/mds.22073

19. Mohammad SS, Fung VS, Grattan-Smith P, et al. Movement disorders in children with anti-NMDAR encephalitis and other autoimmune encephalopathies. Mov Disord. 2014;29(12):15 39-1542. doi:10.1002/mds.25999

20. Dalmau J, Lancaster E, Martinez-Hernandez E, Rosenfeld MR, Balice-Gordon R. Clinical experience and laboratory investigations in patients with anti-NMDAR encephalitis. Lancet Neurol. 2011;10 (1):63-74. doi:10.1016/S1474-4422(10)70253-2

21. Matsumoto L, Yamamoto T, Higashihara M, et al. Severe hypokinesis caused by paraneoplastic anti-Ma2 encephalitis associated with bilateral intratubular germ-cell neoplasm of the testes. Mov Disord. 2007;22(5):728-731. doi:10.1002/mds.21314

22. Vigliani MC, Honnorat J, Antoine JC, et al. Chorea and related movement disorders of paraneoplastic origin: the PNS EuroNetwork experience. J Neurol. 2011;258(11):2058-2068. doi:10.1007/s00415011-6074-1

23. Hou $\mathrm{C}, \mathrm{Wu} \mathrm{W}$, Tian $\mathrm{Y}$, et al. Clinical analysis of anti-NMDAR encephalitis combined with MOG antibody in children. Mult Scler Relat Disord. 2020;42:102018. doi:10.1016/j.msard.2020.102018

24. Hansen N, Ernst L, Rüber T, et al. Pre- and long-term postoperative courses of hippocampus-associated memory impairment in epilepsy patients with antibody-associated limbic encephalitis and selective amygdalohippocampectomy. Epilepsy Behav. 2018;79:93-99. doi:10. 1016/j.yebeh.2017.10.033

25. Hanert A, Rave J, Granert O, et al. Hippocampal dentate gyrus atrophy predicts pattern separation impairment in patients with LGI1 encephalitis. Neuroscience. 2019;400:120-131. doi:10.1016/j. neuroscience.2018.12.046

26. Miller TD, Chong TT, Aimola Davies AM, et al. Focal CA3 hippocampal subfield atrophy following LGI1 VGKC-complex antibody limbic encephalitis. Brain. 2017;140:1212-1219. doi:10.1093/brain/ awx070

27. Finke C, Kopp UA, Scheel M, et al. Functional and structural brain changes in anti-N-methyl-D-aspartate receptor encephalitis. Ann Neurol. 2013;74(2):284-296.

28. Dalmau J, Gultekin SH, Voltz R, et al. Ma1, a novel neuron- and testis-specific protein, is recognized by the serum of patients with paraneoplastic neurological disorders. Brain. 1999;122(1):27-39. doi:10.1093/brain/122.1.27

29. Gultekin SH, Rosenfeld MR, Voltz R, Eichen J, Posner JB, Dalmau J. Paraneoplastic limbic encephalitis: neurological symptoms, immunological findings and tumour association in 50 patients. Brain. 2000;123(7):1481-1494. doi:10.1093/brain/123.7.1481

30. Lövheim H, Norman T, Weidung B, et al. Herpes simplex virus, APOE $\varepsilon 4$, and cognitive decline in old age: results from the betula cohort study. J Alzheimers Dis. 2019;67(1):211-220. doi:10.3233/JAD-171162

31. Wesselingh R, Butzkueven H, Buzzard K, Tarlinton D, O'Brien TJ, Monif M. Seizures in autoimmune encephalitis: kindling the fire. Epilepsia. 2020;61(6):1033-1044. doi:10.1111/epi.16515

32. Fukata Y, Yokoi N, Miyazaki Y, Fukata M. The LGI1-ADAM22 protein complex in synaptic transmission and synaptic disorders. Neurosci Res. 2017;116:39-45. doi:10.1016/j.neures.2016.09.011

33. Yeo T, Chen Z, Yong KP, Wong P, Chai J, Tan K. Distinction between anti-VGKC-complex seropositive patients with and without anti-LGI1/CASPR2 antibodies. J Neurol Sci. 2018;391:64-71. doi:10.1016/j.jns.2018.05.020

34. Panigrahi I, Dhanorkar M, Didel S, Koganti RA. Hunter syndrome with persistent thrombocytopenia. BMJ Case Rep. 2019;12(4): e226518. doi:10.1136/bcr-2018-226518

35. Nishio Y, Kawano Y, Kawada JI, Ito Y, Hara S. A case of refractory cytomegalovirus-related thrombocytopenia that achieved complete remission without antiviral therapy. J Infect Chemother. 2018;24 (12):995-997. doi:10.1016/j.jiac.2018.06.004 
36. Elalfy MS, Nugent D. Viruses, anti-viral therapy, and viral vaccines in children with immune thrombocytopenia. Semin Hematol. 2016;53:S70-2. doi:10.1053/j.seminhematol.2016.04.021

37. Bolis V, Karadedos C, Chiotis I, Chaliasos N, Tsabouri S. Atypical manifestations of Epstein-Barr virus in children: a diagnostic challenge. J Pediatr. 2016;92(2):113-121. doi:10.1016/j.jped.2015.06.007

38. Schulz D, Rothenhöfer C. [The suitability of the Pandy reaction for the rapid evaluation of spinal fluid protein concentrations]. Fortschr Med. 1980;98(22):847-850

39. Lending M, Slobody LB, Mestern J. Cerebrospinal fluid glutamic oxalacetic transaminase and lactic dehydrogenase activities in children with neurologic disorders. $J$ Pediatr. 1964;65:415-421. doi:10.1016/S0022-3476(64)80406-6

40. Bernal F, Graus F, Pifarré A, Saiz A, Benyahia B, Ribalta T. Immunohistochemical analysis of anti-Hu-associated paraneoplastic encephalomyelitis. Acta Neuropathol. 2002;103(5):509-515. doi:10. 1007/s00401-001-0498-0

41. Elishkevitz KP, Nussinovitch U, Nussinovitch M. Lactic dehydrogenase isoenzymes in adolescents with multiple sclerosis. Pediatr Neurol. 2009;41(4):259-262. doi:10.1016/j.pediatrneurol.2009.04.018

42. Cheshenko N, Trepanier JB, Stefanidou M, et al. HSV activates Akt to trigger calcium release and promote viral entry: novel candidate target for treatment and suppression. FASEB J. 2013;27(7):25 84-2599. doi:10.1096/fj.12-220285

43. Cheshenko N, Del Rosario B, Woda C, Marcellino D, Satlin LM, Herold BC. Herpes simplex virus triggers activation of calcium-signaling pathways. J Cell Biol. 2003;163(2):283-293. doi:10. 1083/jcb.200301084
44. Bickler PE, Fahlman CS. Moderate increases in intracellular calcium activate neuroprotective signals in hippocampal neurons. Neuroscience. 2004;127(3):673-683. doi:10.1016/j.neuroscience.200 4.05.035

45. Ayşit-Altuncu N, Ulusoy C, Öztürk G, Tüzün E. Effect of LGI1 antibody-positive $\operatorname{IgG}$ on hippocampal neuron survival: a preliminary study. Neuroreport. 2018;29(11):932-938. doi:10.10 97/WNR.0000000000001055

46. Li Y, Song F, Liu W, Wang Y. Clinical features of nine cases of leucine-rich glioma inactivated 1 protein antibody-associated encephalitis. Acta Neurol Belg. 2020. doi:10.1007/s13760-02001336-Z

47. Zhu F, Shan W, Lv R, Li Z, Wang Q. Clinical characteristics of anti-GABA-B receptor encephalitis. Front Neurol. 2020;11:403. doi:10.3389/fneur.2020.00403

48. Si Z, Wang A, Liu J, Zhang Z, Hu K. Typical clinical and imaging manifestations of encephalitis with anti- $\gamma$-aminobutyric acid $\mathrm{B}$ receptor antibodies: clinical experience and a literature review. Neurol Sci. 2019;40(4):769-777. doi:10.1007/s10072-018-3679-5

49. Ma H, Lundy JD, Cottle EL, et al. Applications of minimally invasive multimodal telemetry for continuous monitoring of brain function and intracranial pressure in macaques with acute viral encephalitis. PLoS One. 2020;15(6):e0232381. doi:10.1371/journal. pone. 0232381
Neuropsychiatric Disease and Treatment

\section{Publish your work in this journal}

Neuropsychiatric Disease and Treatment is an international, peerreviewed journal of clinical therapeutics and pharmacology focusing on concise rapid reporting of clinical or pre-clinical studies on a range of neuropsychiatric and neurological disorders. This journal is indexed on PubMed Central, the 'PsycINFO' database and CAS, and

\section{Dovepress}

is the official journal of The International Neuropsychiatric Association (INA). The manuscript management system is completely online and includes a very quick and fair peer-review system, which is all easy to use. Visit http://www.dovepress.com/testimonials.php to read real quotes from published authors. 